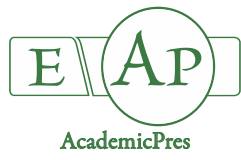

Available online: www.notulaebotanicae.ro

\title{
High-Density Kinetic Analysis of the Metabolomic and Transcriptomic Response of Ginkgo biloba Flavonoids Biosynthesis to Selenium Treatments
}

\author{
Linling $\mathrm{LI}^{1,2,3 a}$, Jie YU ${ }^{1,2 b}$, Honghui YUAN ${ }^{1,2 c}$, Sanxing ZHA ${ }^{1,2,3}$, \\ Kun DENG ${ }^{1,2,3}$, Xian XIAO ${ }^{1,2,3}$, Yanyan $\mathrm{LUO}^{1,2,3}$, Shuiyuan $\mathrm{CHENG}^{1 *}$, \\ Hua $\mathrm{CHENG}^{2,3 * *}$

\begin{abstract}
${ }^{1}$ Wuhan Polytechnic University, College of Biology and Pharmaceutical Engineering, Wuhan, 430023, China; lilinling1437@126.com; 365890655@qq.com;448503382@qq.com; 781634982@qq.com; harry1437@126.com;1814759739@qq.com; 59123371@qq.com;s_y_cheng@sina.com (*correspondingauthor)

${ }^{2}$ Economic Forest Germplasm Improvements and Comprehensive Utilization of Resources of Hubei Key Laboratories, Hubei Huanggang, 438000, China; chenghua1437@126.com (** correspondingauthor)

${ }^{3}$ Hubei Collaborative Innovation Center for the Characteristic Resources Exploitation of Dabie Mountains, Huanggang, 438000, China
\end{abstract} \\ a,b,c These authors contributed equally to this work
}

\begin{abstract}
As one of the rare and precious wood species since the ancient times, Gingko is also known as "living fossil", which is a special plant resource of China. Gingko leaves, containing rich flavonoids, are valued with great medicinal significances. This paper treated Ginkgo seedlings by exogenous Sodium selenite (SS) in two ways: Foliage dressing (FD) and Root application (RA). Then transcriptome sequencing and metabolome test are performed. Results show that external SS has significant influence on the related gene expression level of flavonoids synthesis ways of Gingko, the FD can significantly induce gene expressions as CHS, FLS, FOMT, PAL, MYB1 and MYB2, and RA can significantly induce gene expressions as FOMT, MYB1 and MYB2. Compared with the control group, FA selenium application can help to accumulation of flavonoids, flavonols, flavonoids-C and isoflavones, especially quercetin and kaempferol that had a remarkable increase. This proved that a proper concentration of inorganic SS could promote the synthesis and accumulation of flavonoids in Gingko. qRT-PCR analysis also depicts that leaves treatment of sodium selenite can remarkably enhance the gene expression of CHS, FLS, FOMT and PAL, and RA selenium application can induce the gene expression of FLS and FOMT, but restrain the gene expression of CHS and PAL. Through the ways of FD and RA selenium application, this paper basically studied the regulatory effect of SS on ginkgo flavonoids synthesis and has laid a theoretical basis to improve flavonoids content in Ginkgo leaves through cultivation control means.
\end{abstract}

Keywords: flavonoids; foliage dressing; Gingko biloba; metabolome; sodium selenite; transcriptome

\section{Introduction}

Selenium (Se) is one of the indispensable nutrient elements to humans and poultry, but $2 / 3$ of the world lacks it (Schiavon et al., 2013). Although Se is a known anticarcinogen, little is known regarding how Se affects other nutritional qualities in plants. Some documents show that the se-biofortified fruits enhance the levels of antioxidant flavonoids naringenin chalcone and kaempferol and a concomitant decrease of cinnamic acid
derivatives(Schiavon et al., 2013). In buckwheat, treatment with Se, the content and accumulation of flavonoids and rutin in all organs were enhanced (Tian and Wang, 2008).

Flavonoids are ubiquitous plant secondary products that have diverse functions in plant physiology and ecology, including protection against UV-B radiation and pathogen attack, attraction of pollinating insects, regulation of male fertility, cell cycle regulation, auxin transport, and the also possess health beneficial properties (Khan et al., 2010; Cheng et al., 2011; Xu et al., 2014b). Flavonoid biosynthesis has been well documented various plants. Phenylalanine, as

Received: 03 Jan 2019. Received in revised form: 29 May 2019. Accepted: 31 May 2019. Published online: 06 Jun 2019. 
the common precursor, can be catalyzed to different types of flavonoid compounds via different biosynthesis pathways. Most enzymes involved in these pathways have been well investigated. To be more precise, phenylalanine ammonia lyase, cinnamate 4-hydroxylase and 4-coumarate: CoA ligase are involved in the general phenylpropanoid pathway; chalcone synthase, chalcone isomerase, and flavanone 3hydroxylase are early biosynthetic genes; dihydroflavonol 4reductase is involved in the proanthocyanidin and anthocyanin pathways; UDP-glucose:flavonoid 3glucosyltransferase (UFGT) is involved in the anthocyanin and flavonol pathways; anthocyanin synthase (ANS) is involved in the anthocyanin and PA pathways; flavonol synthase (FLS) is specifically involved in the flavonol pathway; and anthocyanin reductase (ANR) is specifically involved in the PA pathway (Fischer et al., 2007; Zhai et al., 2016).

Ginkgo biloba is often called "a living fossil" of the plant kingdom, and has been well studied owing to the many active ingredients, such as flavonoids, contained in its leaves. At present, about 38 different flavonoids, especially, multiform glycosides of flavonols, have been isolated from G. biloba (Bilia, 2002; Beek and Montoro, 2009). However, the concentrations of these economically important compounds are often insufficient to justify commercial exploitation of Ginkgo leaves. In recent years, a number of investigations have focused on the biological mechanism of flavonoid formation, the vitro chemical synthesis of flavonoids, and the use of tissue culture for producing flavonoids, among genetic modification aspects in Ginkgo (Pang et al., 2005; Hua et al., 2013; Li et al., 2014).

Here we report the potential for increasing Se and flavonoids content in G. biloba leaves through foliar or root supplementation of selenite. We tested the transcriptome and metabolome change by a different fertilization approach by adding selenite to $G$. biloba. We also discuss the gene CHS, PAL, FLS, FOMT expression and its potential function in secondary metabolism of Ginkgo. This could also reveal the effecting of selenite in improving flavonoids contents in G. biloba.

\section{Materials and Methods}

Plant materials and treatments

Two-years old of grafted G. biloba seedlings were growing in a greenhouse in Huanggang (E, $111^{\circ} 54^{\prime}-112^{\circ} 19^{\prime}$, $\mathrm{N}, 30^{\circ} 6^{\prime}-30^{\circ} 39^{\prime}$, Hubei province, central of China) were sampled as plant materials. For gene cloning and tissue expression, diverse tissues including young leaves, mature leaves, ovules, stamens, albumen, gynoecia, stems and roots were collected for DNA and RNA extraction as described by Cheng (2013a).

Two-years old cuttings from the same genotypic strain of $G$. biloba were subjected to treatments with $10 \mathrm{mg} / \mathrm{ml}$ sodium selenite, seedlings were treated with $\mathrm{A}$ method (spraying on leaves) and B method (irrigating on roots). The sodium selenite were dissolved in $0.01 \%$ Tween 20 and sprayed onto young leaves. The control leaves were sprayed with an equivalent volume of $0.01 \%(\mathrm{v} / \mathrm{v})$ Tween 20 . All samples were immediately frozen in liquid nitrogen and stored at $-80{ }^{\circ} \mathrm{C}$ prior to total $\mathrm{RNA}$ extraction, transcriptome sequencing, and expression analysis.

Total RNA extraction and transcriptome sequencing

The total mRNA was enriched with magnetic beads coated with oligo dTn, and then fragmented, which was reverse transcribed to first-stranded cDNA. Doublestranded cDNA was synthesized with random hexamer primers, purified and added with sequencing linkers. Fragments of the correct size were purified with Universal DNA Purification Kit, and the sequencing libraries were prepared with the RNA-Seq Library Construction Kit. The quality and quantity of the libraries were verified using an Agilent 2100 Bioanalyzer and ABI real time RT-PCR. The qualified cDNA libraries were used for paired-end sequencing on an Illumina HiSeq 2500 platform.

\section{De novo transcriptome assembly and annotation}

After removing those reads with only adaptor and unknown nucleotides $>5 \%$, or those that were of low quality, the clean reads were filtered from the raw reads. The clean reads were then assembled de novo using the Trinity platform (http://trinityrnaseq.sourceforge.net/) with the parameters of ' $\mathrm{K}$-mer $=25$, group pairs distance $=300$ ' (Grabherr et al., 2011). For each library, short reads were first assembled into longer contigs based on their overlap regions. Then different contigs from another transcript and their distance were further recognized by mapping clean reads back to the corresponding contigs based on their paired-end information, and thus the sequence of the transcripts was produced. Finally, the potential transcript sequences were clustered using the TGI Clustering tool to obtain uni-transcripts (Qian et al., 2014). Uni-transcripts were aligned to a series of protein databases using BLASTx (E-value $\leq 10-5)$, including the NCBI non-redundant $(\mathrm{Nr})$, the Swiss-Prot, the Trembl, the Kyoto Encyclopedia of Genes and Genomes (KEGG) (http:// www. Genome .jp/kegg/kegg2.html), and gene ontology (http:/ / wego. genomics. org.cn/cgi-bin/ wego/index.pl) databases. To determine the gene coverage, the reference sequences for all three color-related pathways were downloaded from the public databases. All isoforms of all color-related genes present in the databases examined were aligned against corresponding reference sequences using BLASTx. The deduced amino acid sequences of uni-transcripts were required to be longer than $70 \%$ of the corresponding sequences. If a uni-transcript met the criteria, it was assumed to contain a near full-length contig. If not, targeted assembly was performed to obtain even greater coverage of the respective genes. All reads in the databases examined were mapped to the reference sequences and the mapped reads were then assembled using clustering and CAP3 assembly (http://compbio.dfci.harvard.edu/tgi/software/).

\section{Gene expression analysis}

Analysis RT-PCR was performed to measure the relative transcript levels of selected genes. First-strand cDNA synthesis was conducted in triplicate for each sample according to the manufacturer's instructions (PrimeScript ${ }^{\mathrm{TM}}$ RT Reagent Kit, TaKaRa, Dalian, China). The house keeping gene, namely glyceraldehyde- 3-phospate dehydrogenase gene (GbGAPDH, L26924), was used as a reference gene with primers of GAPU and GAPD. Genespecific primers of flavonoids were list in Table 1 . The 
794

experiments were repeated independently with three biological replicates using SYBR $^{\circ}$ Premix Ex Taq ${ }^{\mathrm{Tx}}$ II (TaKaRa, Dalian, China) in the Applied. Biosystems 7500 Realtime PCR systems (Foster City, CA, USA). Relative expression level was calculated according to the method described by Schmittgen (2008).

\section{LC-MS/MS analysis of flavonoids}

Samples were analyzed using LC-ESI-Q TRAP-MS/MS and quantification of flavonoids was carried out in the multiple reaction monitoring (MRM) mode. The analytical conditions were as described by Chen et al. (2013). Data acquisition, peak integration, and calculations were performed by using Analyst 1.5 software (Metaware, Wuhan China). Flavonoids were quantified by calculating the area of each individual peak and comparing this to standard curves obtained from flavonoid authentic standards, including apigenin 6-C-glucoside, apigenin 7-Oglucoside, luteolin 7-O-glucoside, quercetin 3-O-glucoside, eriodictyol 7-Oglucoside, naringenin 7-O-glucoside, and kaempferol 3-Oglucoside. Calibration curves were drawn by plotting at four different concentrations of each flavonoid standard according to the peak area.

\section{Data annotation and statistical analysis}

Raw sequence reads were filtered by the Illumina pipeline. Then the clean reads were subjected to the TopHat2-Cufflinks-Cuffmerge-Cuffdiff standard pipeline for identification of DEGs. These DEGs were blasted against the GO, KEGG, and COG of proteins database for enrichment analysis (Jie et al., 2015).

Metabolite data were $\log 2$-transformed to improve normality and normalized. A total of 91 and 68 flavonoids have been used for hierarchical clustering analysis by Wuhan metaware (http://www.metware.cn/) to study flavonoid tissue specific accumulation and natural variation. Association analysis between flavonoid accumulation and gene allelic status was performed using Student's ttest. SNPs that showed significant variation with various flavonoids $(\mathrm{P}<0.01)$ were used for further analysis. The experimental results were expressed as mean \pm standard error (SE) of three replicates. The data were analyzed using Statistical
Product and Service Solutions (SPSS, Version 17.0) at $\mathrm{P}<$ 0.05 (Fisher's protected least significant difference).

\section{Results}

\section{RNA-Seq and assembly}

To understand the molecular basis of selium treatment in Ginkgo leaves and roots, the treatments sample and control were used to build three libraries for highthroughput sequencing. The three libraries (A, B, and CK) produced about 447207439 raw reads and 431640524 clean reads, respectively, the average GC contents is about $47.64 \%$, and the Q20 percentages is $97.8 \%$ (Table 2). All sequences were assembled to give 142834 unigenes and 93696497 nucleotides with a mean length of $656 \mathrm{bp}$ (Table 3 and Table 4).

\section{Functional classification by $G O$ and $C O G$}

Functional annotations of unigenes $\mathrm{GO}$ is a useful program for annotating and analyzing the functional categorization of annotated genes. To facilitate the organization of the cranberry transcripts into putative functional groups, GO terms were assigned using Blast GO. A total of 268,025 unigenes were assigned into GO ontologies based on their similarity to sequences with previously known functions, including 53,869 sequences assigned to the molecular function category 128,805 to the biological process category and 85,351 to the cellular component category (Fig.1). In these results, about 114 genes were classified to flavonoid metabolic process.

The KOG database can phylogenetically predict and classify the completeness of the unigene sequences. Based on sequence homology 18,671 unigenes $(13.07 \%$ of all the unigenes) were assigned into $26 \mathrm{KOG}$ categories (Fig. 2).

KEGG pathway mapping KEGG analysis can help us further understand the specific processes, gene functions and gene interactions at a transcriptome level (Fig. 3). In our study, 18671 all unigenes were mapped to 131 predicted metabolic pathways through the KEGG database.

The largest category was metabolism (15,776) which included biosynthesis of amino acid metabolism $(2,326)$,

Table 1. The gene clone and analysis primers of GbIRL1

\begin{tabular}{ccc}
\hline Primer & Sequence $\left(5^{\prime}-3^{\prime}\right)$ & Description \\
\hline CHSU & GGCAATCGGCACTGCTACTC & GbCHS Primer for qRT-PCR, forward \\
CHSD & TCTTGTGCTCGCTGTTGGTAA & GbCHS Primer for qRT-PCR, reverse \\
FLSD & CATCTCTCCCTCGCTCTTCG & GbCHS Primer for qRT-PCR, forward \\
FOMT1 & AGAACTGCTTGCCAACCGTC & GbCHS Primer for qRT-PCR, reverse \\
FOMT2 & AGAGTGTTAGCCAGCCATTCAG & GbCHS Primer for qRT-PCR, forward \\
PAL1 & CTTCCCATTCTCATCTGTGGTTAC & GbCHS Primer for qRT-PCR, reverse \\
PAL2 & AGGTGTGTTGTTCCGCATCC & GbCHS Primer for qRT-PCR, forward \\
MYB1U & CCACCTTTGAAATGGATGCG & GbCHS Primer for qRT-PCR, reverse \\
MYB1D & GGTGGTCTATCATTGCTGCGC & GbCHS Primer for qRT-PCR, forward \\
MYB2U & GACGACGAGTTTGCTGATGATC & GbCHS Primer for qRT-PCR, reverse \\
GAPU & GTCAGTTGTGGATTGTGGGTTT & GbCHS Primer for qRT-PCR, forward \\
GAPD & TTCTACCAGTTGATGAAGTGCT & GbCHS Primer for qRT-PCR, reverse \\
\hline TYB & TAGGAATCCCGAGGAAATACC & Used as control gene for qRT-PCR, forward \\
\hline
\end{tabular}

Note: Restriction site sequences are indicated in italic letter and underline. 
Table 2. Overview of the sequence data and output quality

\begin{tabular}{ccccccc}
\hline Sample & Raw Reads & Clean reads & Error $(\%)$ & Q20 (\%) & Q30 (\%) & GC (\%) \\
\hline CK1 & 47264688 & 45717488 & 0.01 & 97.72 & 94.17 & 47.11 \\
CK2 & 53143152 & 50777376 & 0.01 & 97.97 & 94.70 & 46.64 \\
CK3 & 54498866 & 52759210 & 0.01 & 97.84 & 94.43 & 46.53 \\
A1 & 48952556 & 47293550 & 0.01 & 97.78 & 94.29 & 48.59 \\
A2 & 51089744 & 49200524 & 0.01 & 97.69 & 94.09 & 48.15 \\
A3 & 48040934 & 46329816 & 0.01 & 97.87 & 94.47 & 48.71 \\
B1 & 48347886 & 46791426 & 0.01 & 97.73 & 94.19 & 48.18 \\
B2 & 50454236 & 48897574 & 0.01 & 97.86 & 94.45 & 47.37 \\
B3 & 45412272 & 43873542 & 0.01 & 97.72 & 94.14 & 47.47 \\
\hline
\end{tabular}

Table 3. The frequency distribution list of transcript sequence

\begin{tabular}{cccccc}
\hline Transcript length interval & $200-500 \mathrm{bp}$ & $500-1 \mathrm{kbp}$ & $1 \mathrm{k}-2 \mathrm{kbp}$ & $>2 \mathrm{kbp}$ & Total \\
\hline Number of transcripts & 129245 & 39899 & 38220 & 35020 & 242384 \\
Number of unigenes & 100266 & 19949 & 12258 & 10361 & 142834 \\
\hline
\end{tabular}

Table 4. Overview of the distribution of splicing length

\begin{tabular}{cccccccc}
\hline & Min Length & Mean Length & Median Length & Max Length & N50 & N90 & Total Nucleotides \\
\hline Transcripts & 201 & 971 & 449 & 21317 & 1920 & 333 & 235330603 \\
Unigenes & 201 & 656 & 317 & 21317 & 1215 & 253 & 93696497 \\
\hline
\end{tabular}

biosynthesis of other secondary metabolites (786), carbohydrate metabolism (3884), energy metabolism (1,371), glycan biosynthesis and metabolism (358), lipid metabolism (1,594), metabolism of cofactors and vitamins (6,360), metabolism of terpenoids and polyketides (527), flavonoid and flavone biosynthesis (99), and other subcategories. These results suggest that the secondary metabolic processes are active pathways in Ginkgo flavonoid and anthocyanin biosynthesis after Se treatment.

To identify DEGs among SS treatment samples, we compared them with each other and identified unigenes that were at least 2-fold up or down regulated between the three samples, with p-value less than 0.05. Then, hierarchical clustering was used to gain a global view of DEGs. The DEGs analysis of the A was similar to the $\mathrm{B}$ treated samples. In total, 4,653 different genes were identified between A and control, of which, up-regulated gene was 2,934, down-regulated was 1,719; about 2,546 different genes were identified between $B$ and control, up was 1,920, down was 626; between $A$ and B, 394 genes were identified, up-regulated was 244 , down regulated was 150 genes respectively (Fig 4). From the DEGs analysis results, it was clear that $\mathrm{Se}$ has a significant impact on the transcriptome of Ginkgo leaves. However, it also could be deduced from the heat map that the $\mathrm{A}$ and $\mathrm{B}$ sample have a different transcriptome.

Gene Function Classification (GO)

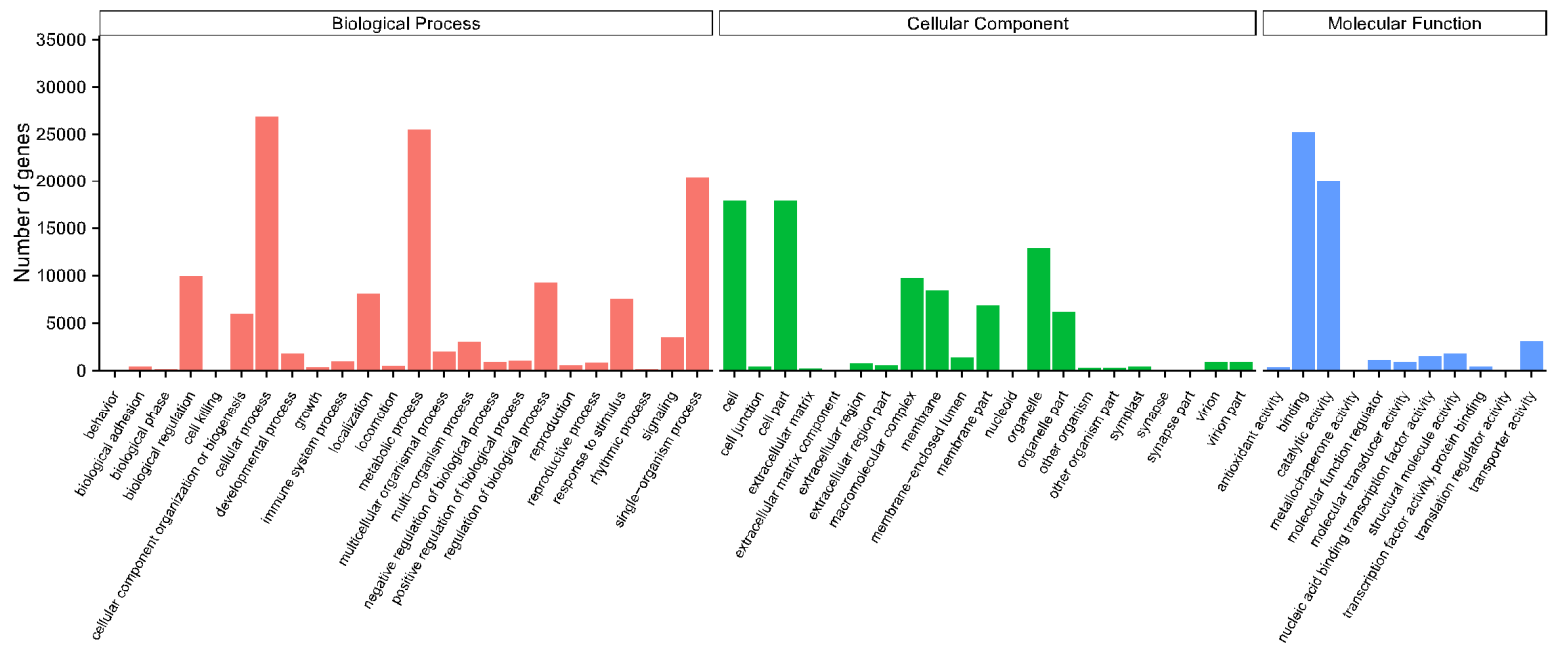

Fig. 1. Histogram of GO analysis of all-unigenes 
796

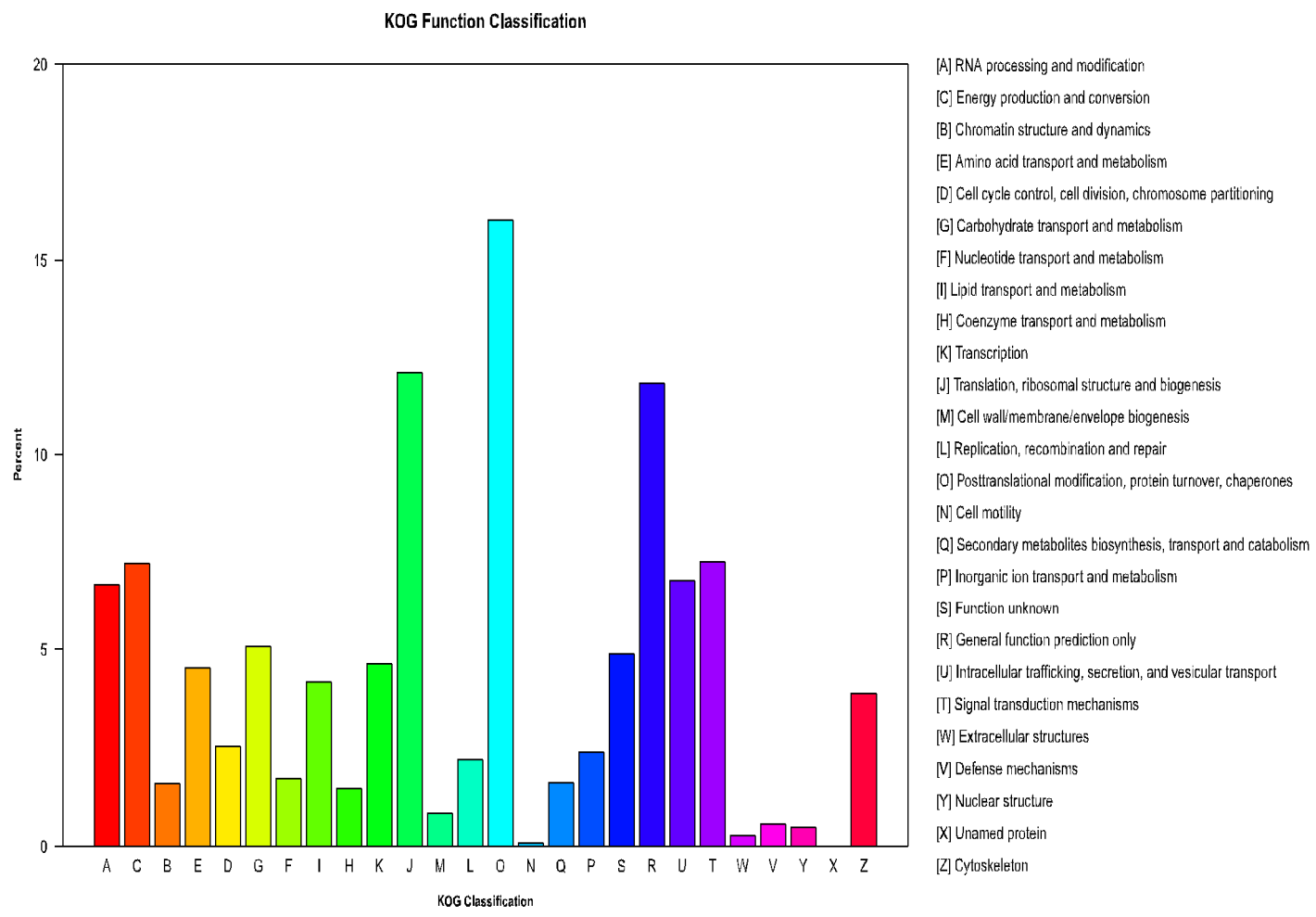

Fig. 2. Histogram of KOG classification of all-unigenes

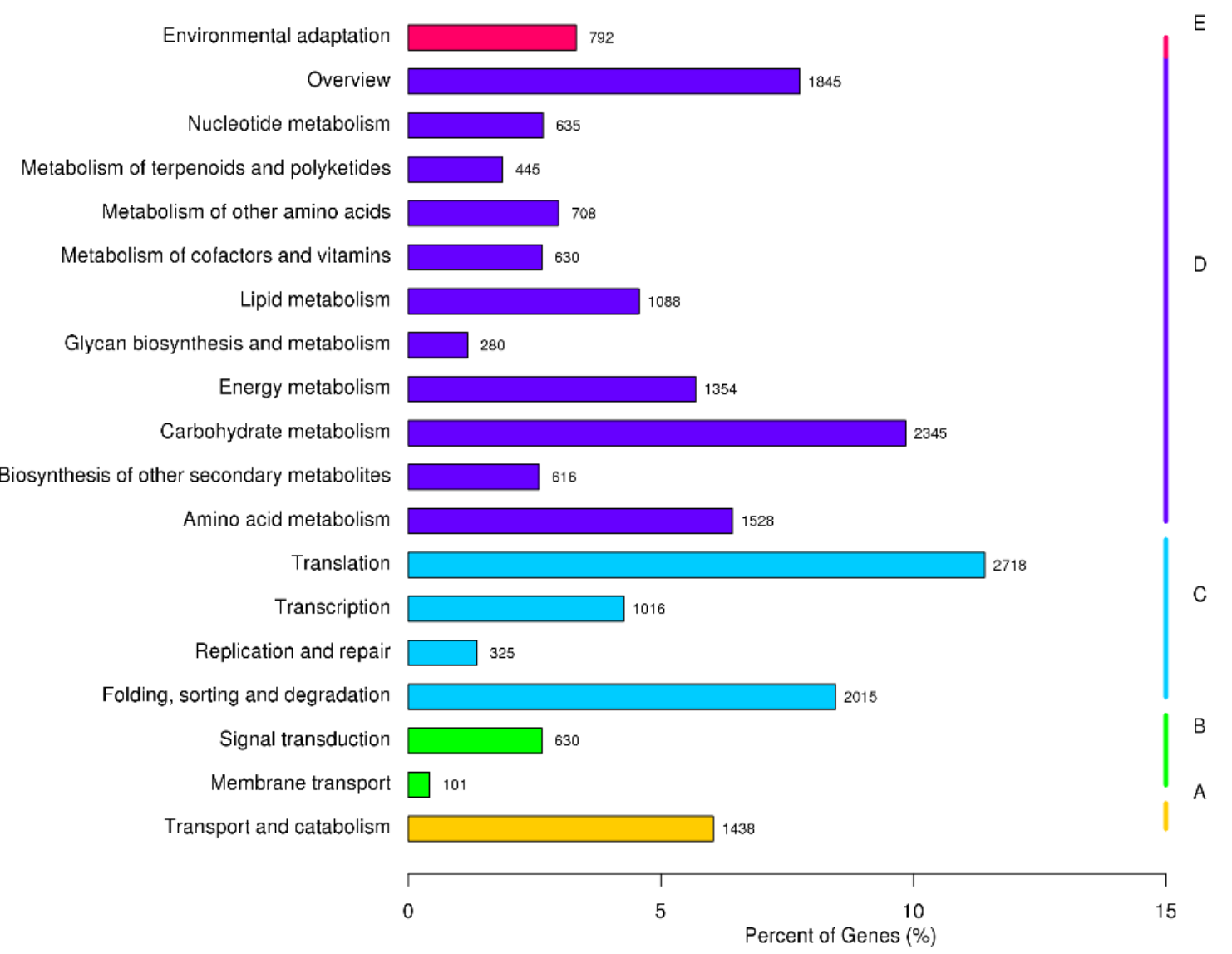

Fig. 3. The KEGG classification histone after Ginkgo treatment with Se 

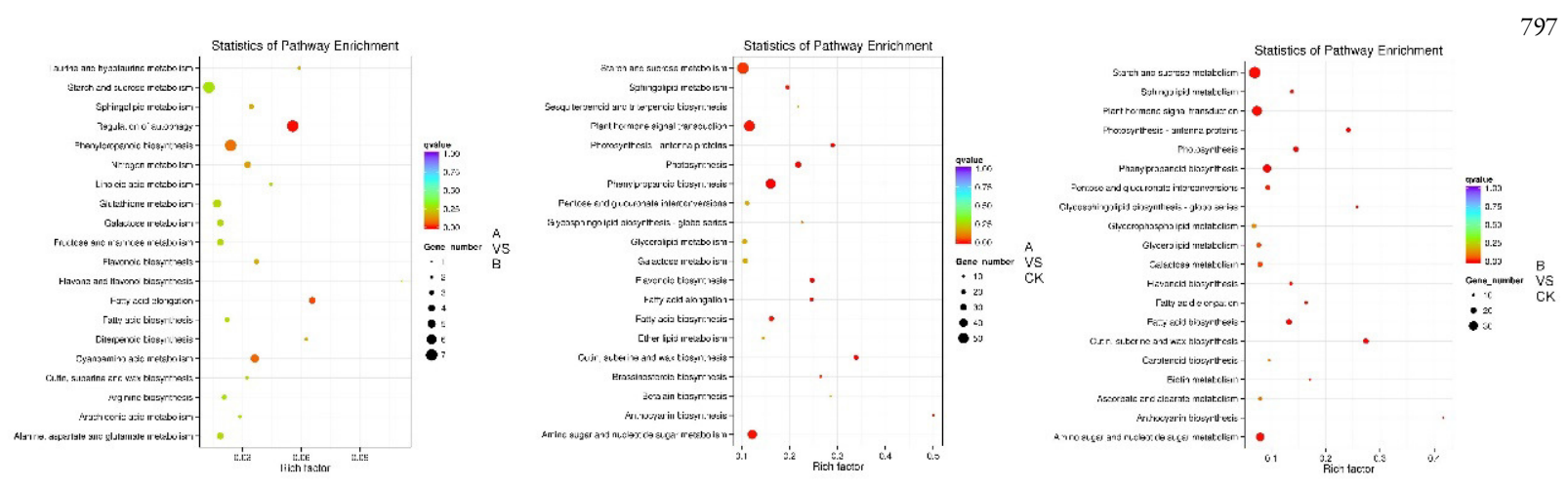

Fig. 4. The statistics of KEGG pathway scatterplot analysis of all-unigenes

Transcriptome analysis of key genes in flavonoids

According to the cluster analysis of differentially expressed genes and KEGG enrichment analysis (Fig. 5), CHS genes are concentrated in flavonoids biosynthesis (ko00941) pathway and circadian rhythm plant (ko04712) path. Compared with the control group, treatment group A has significant up-regulated genes in flavonoids biosynthesis path, but no changes are seen in circadian rhythm plant path. No obviously changes are detected in treatment group B compared with control group. FLS genes are also concentrated in flavonoids biosynthesis (ko00941) path. Only treatment group A has a significant up-regulation compared with control group. No expression changes are detected in treatment group B compared with the control group. FOMT genes are concentrated in phenylpropanoid biosynthesis (ko00940), which has a significant upregulation in treatment $\mathrm{A}$, but no significant changes are seen in treatment $\mathrm{B}$ compared with the control group. PAL genes are concentrated in phenylpropanoid biosynthesis (ko00940) path and phenylalanine metabolism (ko00360) path. In phenylpropanoid biosynthesis, treatment group A has an obvious up-regulation, while treatment group B has no significant change compared with the control group. This shows that $S S$ has the effect to promote the expression of CHS, FLS, FOMT and Pal genes. The difference between the two treatment groups shows that different treatment means have different promoting effect on these genes in ginkgo leaves. The sensitivity of leaves on the external inorganic SS is stronger than that of the root. Hence, SS application on the leaves will receive a faster response.

\section{Screening results of significant differential metabolites}

Based on self-built database and existing mass spectrum public database (e.g. MassBank, KNAPSAcK, HMDB, MoTo DB and METLIN, etc.), qualitative and quantitative analysis is performed on the first-order mass spectrum and second order mass spectrum detected. The experiment tested totally 991 known and unknown metabolites. Fig. 6 shows the multimodal test figure of MRM (multiple reaction monitoring) metabolites, which presented the maters that were detected in the samples. Each color represents a type of metabolite. Fig 7 shows the volcano map of metabolites contents in different Se treatment sample.

After the screening of flavonoids differential metabolites, totally 315 differential metabolites were found between the control group and treatment group A, 324 differential metabolites were found between the control and treatment $\mathrm{B}$, while only 154 differential metabolites were found between treatment $A$ and treatment $B$ (Fig. 8).

Totally 253 types of flavonoids and flavanone matters were teste, including 21 types of anthocyanins, 98 types of flavonoids, 50 types of flavonoids-C, 37 types of flavonols, 3 types of flavonoe-lignan, 34 types of flavanones, and 10 types of isoflavones.

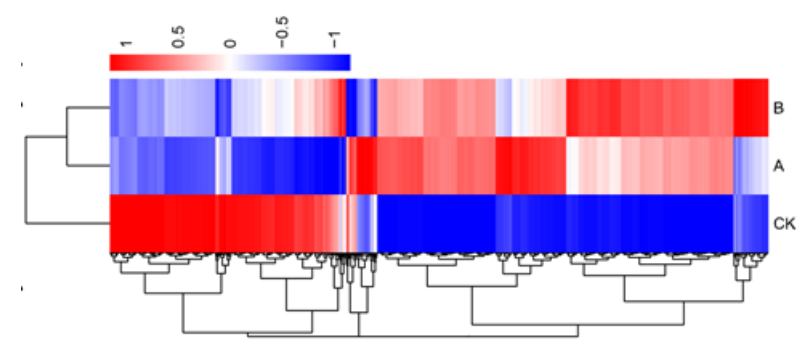

Fig. 5. Cluster analysis of differentially expressed genes during Se treatment

Expression changes and cluster analysis of 13452 genes that were differentially expressed between any two of three samples. Each row represents a differentially expressed gene, while each column represents a sample. Changes in expression levels are shown in color scales with saturation at 2.0-fold changes. Green and red color gradients indicate a $\log 10(\mathrm{FPKM}+1)$ decrease and increase in transcript abundance, respectively

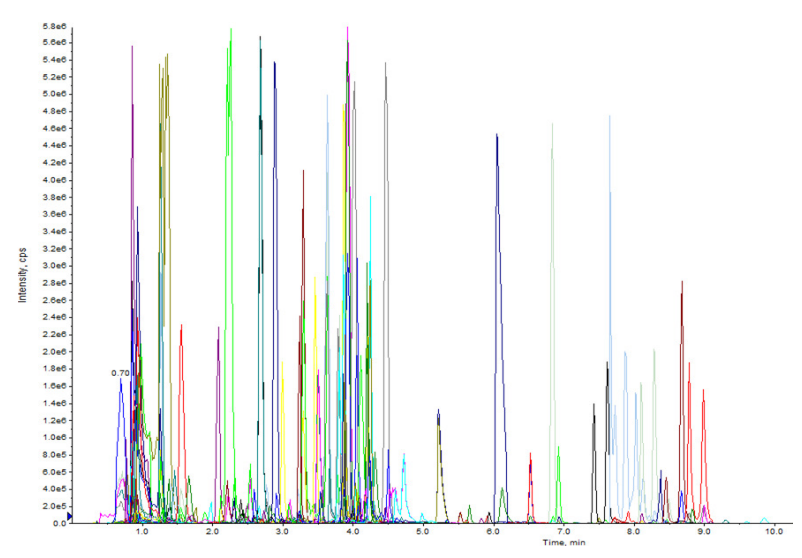

Fig. 6. Metabolite detection multimodal map 

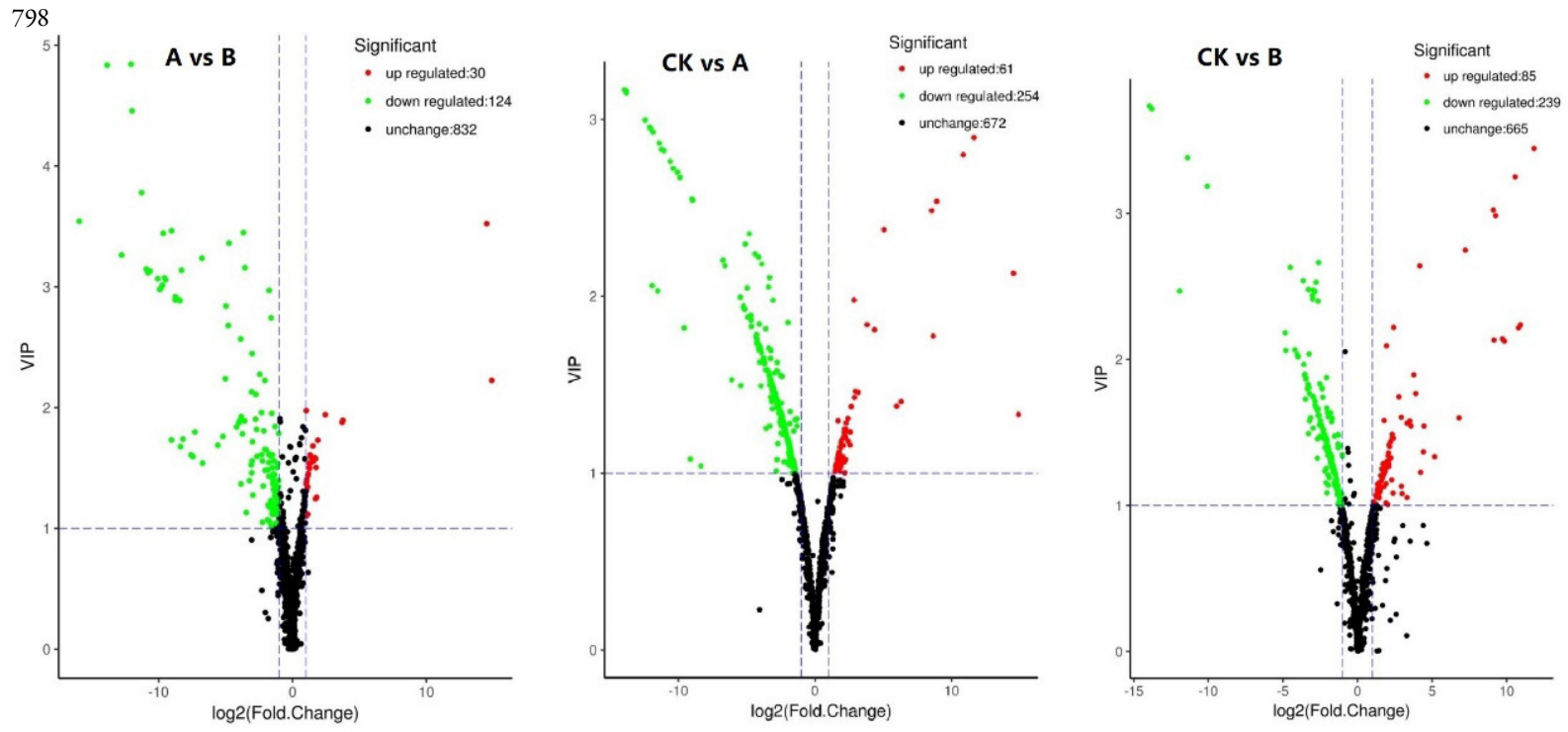

Fig. 7. The volcano map of metabolites contents in different Se treatment sample. Each point in the graph represents a metabolite; the green indicated differential down regulated metabolites, red was the metabolite of up regulated and black indicated the no differential metabolites. A is treatment of foliage dressing; $\mathrm{B}$ is the root application

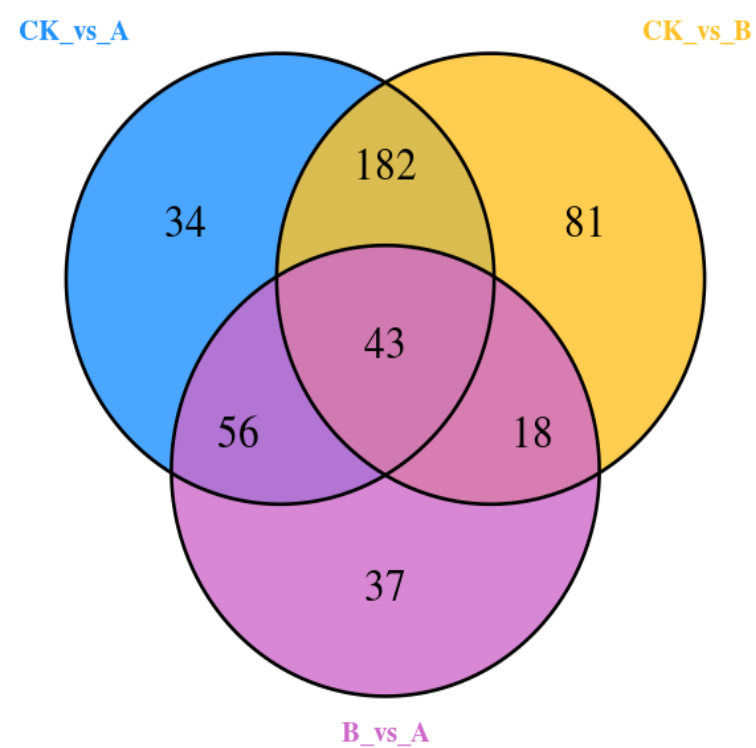

Fig. 8. Wayne diagram of different groups of metabolites

\section{Metabolome analysis of key genes in flavonoids}

In the 253 flavonoids differential metabolites tested in the metabolome, the content difference is significant between group A, group B and the control group (Fig. 9a). Analytical data shows that the flavonoid matters (e.g. proanthocyanidins, flavonoids-isoflavone, flavonoids, flavonoe-lignans, flavonols, flavonoids-C, flavones, anthocyanins) in the treatment group $\mathrm{A}$ had a remarkable up-regulation compared with the control group, especially the flavonoids, flavonols, and flavonoe-lignans. However, the content of proanthocyanidins and flavonoid-isoflavones in treatment group $\mathrm{B}$ has a certain down-regulation compared with the control group, and there are no significant changes of flavonoid metabolites between group $A$ and group B. The flavonoid matters as flavonoidsisoflavones, flavonoids, flavonoe-lignans, flavonols and flavonoid-C in Group A all have a certain level of upregulation.

Based on the transcriptome data, four critical genes (CHS, FLS, FOMT and PAL) in flavone metabolism and two MYB transcription factors connected with flavone metabolism were selected to perform the differential analysis of expression (Fig. 9b). According to the analysis results, the expression level of PAL, MYB1 and MYB2 in $\mathrm{CK}$ is relatively low. The PAL expression level in group $\mathrm{B}$ is similar to the control group, while group $\mathrm{A}$ has a quite high PAL expression level. The FOMT expression level in group $A$ is increased significantly. Group A has the same expression level of MYB2 with group $\mathrm{B}$. The expression level of MYB1 in group A is a little more than group B. The expression level of CHS and FLS is a little higher than CK in both group A and group B. This means that SS can upregulate the expression level of CHS, FLS, MYB1 and MYB2. Compared with group B, FD application can significantly up-regulate the expression level of FOMT and PA1, compared with group B. The metabolome results show that the metabolic rate of flavonoids, flavonols and flavone- $\mathrm{C}$, isoflavones in group $\mathrm{A}$ is apparently higher than $\mathrm{B}$ and $\mathrm{CK}$, and the metabolic rate of procyanidins in group $A$ and group $B$ is apparently lower than CK. This demonstrates that SS may restrain the accumulation of procyanidins, and FD application might be helpful in the accumulation of flavonoids, flavonol, flavone- $\mathrm{C}$ and isoflavone.

The correlation analysis results showed, the four genes, CHS, FLS, FOMT and PAL can be associated with most flavonoids (Fig. 10). CHS may associated with the metabolism of Chrysoeriol, Astragalin, chrysoeriol, Isorhamnetin, Quercitrin, apigenin and luteolin; FLS is 
the same as CHS, also associated Chrysin O-hexoside and Kaempferol. PAL may associated with Chrysoeriol, Avicularin, Tricin 5-O-rutinoside, Trifolin, Quercetin, Apigenin, Luteolin and Baicalein; FOMT suggest associating with Tricin, Kaempferol-3-O-galactoside, Apigenin 7-O-glucoside, Luteolin and Syringetin-3-Ogalactoside.

The four genes can also associate with Anthocyanins product in Ginkgo leaves after treatment with Se. CHS and PAL deduced to associate with metabolism of Cyanidin 3glucoside and Delphinidin, FLS with Peonidin 3-Glu; FOMT with Delphinidin.

Isoflavones correlation analysis results showed, CHS be associated with isotrifoliin, isohemiphloin, pentamethoxychalcone. Besides, FLS may associate with isotrifoliin, isohemiphloin, amentoflavone and calycosin. PAL correlate with amentoflavone, calycosin. FOMT affected the product of calycosin and phloretin.

In the metabolism of flavonol analysis, CHS and FLS gene regulated the metabolism of avicularin and kaempferol 7-O-rhamnoside in Ginkgo leaves; PAL participates in the metabolism of resokaempferol 7-O-hexoside; FOMT expression level regulates resokaempferol 7-O-hexoside and hyperin. FLS gene regulated the catechin metabolism, as catechin and epigallate eatechin gallate, FOMT and PAL associated with protocatechuic acid.

\section{The expression analysis of flavonoids synthesis gene}

To obtain accurate data of CHS, FLS, FOM, and PAL expression level and to determine whether they are influenced by exogenous SS we treated Ginkgo's leaves and roots with SS solution in the same concentration. The first sampling time happened one week after the treatment; samples are taken every one week; six batches of samples were obtained totally. The expression level of the above 4 genes were then verified through fluorescent quantification PCR (Fig. 11).

Similar to transcriptome data, the transcriptional level of the four genes in group A after exogenous FD treatment had obvious increase compared with the control group, while there are no significant differences between RA and the control group. Besides, CHS and PAL gene had a small decline. It can be seen that Se element exerts different influence on leaves and roots.

According to transcription analysis, the expression level of CHS in leaves had a small increase at the sampling point one week after the treatment. This level rose to its peak at the second week, which was 19 times larger than the control group; it declined at the third week and returned to the control level at the fourth and fifth week. As for the roots, the expression level of CHS at the sampling point one week after the treatment dropped to $10 \%$ of the control group. This level maintained until the sampling point of the second week and then slightly increased to $40 \%$ of the control group; this level maintained to the fifth week and eventually decreased to $20 \%$ of the control group at the sampling point of the sixth week.

As for FLS, the expression level in leaves had a slight increase at the sampling point one week after the treatment, which then increased sharply to 12 times as much as that of the control group. It dropped a little at the third week and then reached its peak at the fourth week, about 13 times of the control group; the expression level declined largely at the sampling point of the fifth week, equaling to the level of the control group; it stabilized until the last sampling in the sixth week. FLS's expression level at the first sampling reduced to $50 \%$ of the control group and experienced slight drop during the second week; the level rose and peaked (about 9 times of the control group) in the third and fourth week, which then fell to normal.

The analytical results of PAL's expression level in leaves showed a slight increase the first week after treatment. But it declined in the second week and dropped to a level lower than the control group. In the fourth week, the expression level had a sharp increase but then decreased to its valley in the fifth week, only about $20 \%$ of the control group. The value rocketed to the peak in the sixth week, approximately 30 times of the control group. PAL's expression level in roots experienced a slight increase the first week after SS treatment, but then fell to the level of the control group in the second week. It dropped to $20 \%$ of the control group in the third week and maintained this status until the last sampling.

The expression level of FOMT in leaves had a sharp increase at the sampling point the first week after treatment, which was 37 times of the control group; it grew in the second week and then decreased in the third one, but the level was still 27 times of the control group. In the fourth week, the expression level rocketed to the peak-57 times of the control group, which then declined and maintained at a level 6 times of the control group. FOMT's expression level in roots after treatment also increased in the first week, which was about 19 times of the control group; it experienced small declines in the second and third week, then it rose a little in the fourth week. It finally dropped to $30 \%$ of the control group in the sixth week.

\section{Discussion}

In recent years, the importance of Se in the human diet has received great attention worldwide because the effects of Se deficiency on human health have become a topic of interest in public health systems (Thavarajah et al., 2008). Flavonoids are ubiquitous plant secondary products that have diverse functions in plant physiology and ecology (Winkel-Shirley, 2002). Flavonoids also have many pharmaceutical uses in human health, with pharmacological properties that have been especially valuable in the treatment of early-stage Alzheimer's disease, vascular dementia, cancer and many other diseases (Kennedy and Wightman, 2011). Flavonoids are synthesized by the wellstudied phenylpropanoid pathway and most of the enzymes and genes involved in flavonoid biosynthesis have been characterized (Cheng et al., 2011; Cheng et al., 2013a; Cheng et al., 2013b; Ye et al., 2016).

\section{Influence of Se on Gingko's secondary metabolites}

Currently, it is widely accepted that Se has a dual effect on the growth of plants. Se in proper quantity can stimulate the growth of plants, improving both their quality and yield. But excessive Se would suppress the plants' growth. In tomato, the Se-biofortified fruits showed enhanced levels of the antioxidant flavonoids, naringenin, chalcone and 


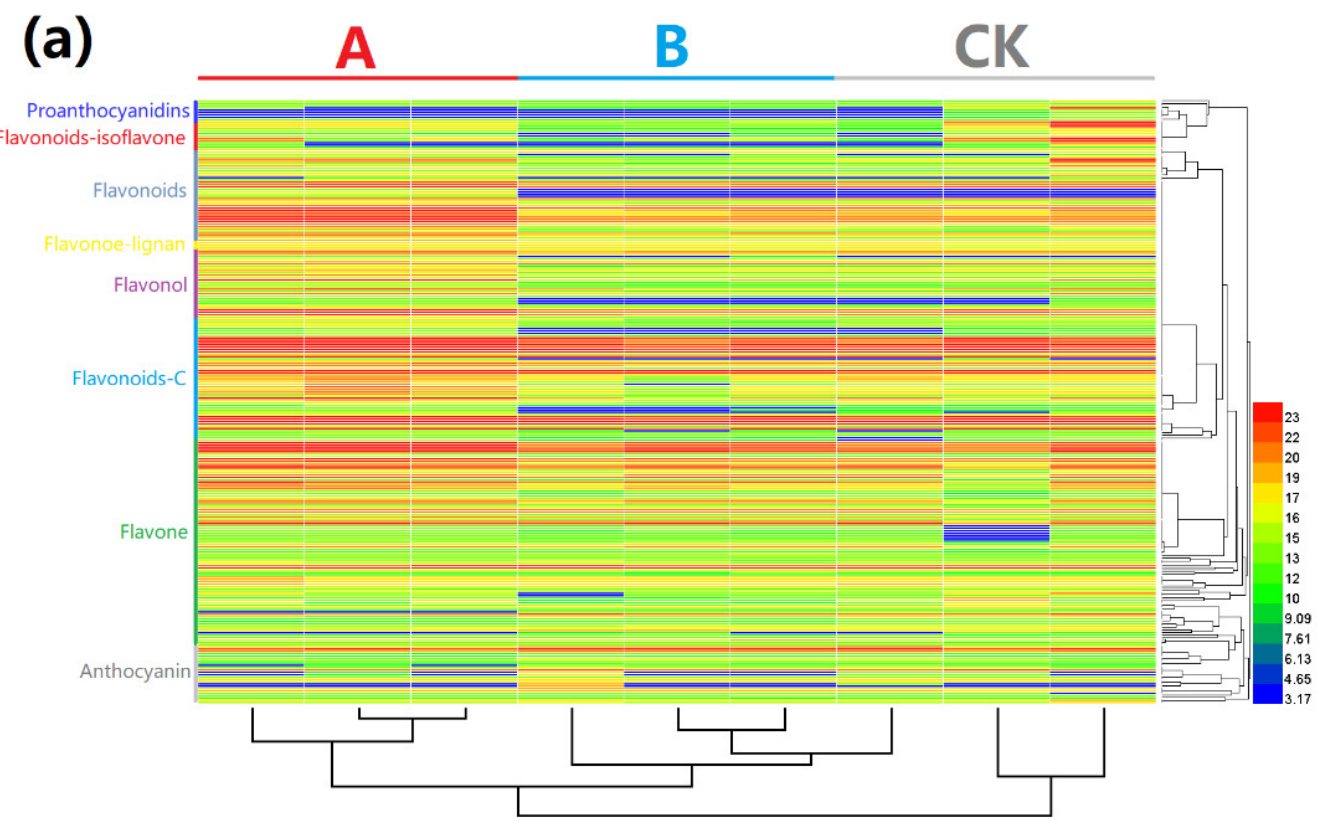

(b)

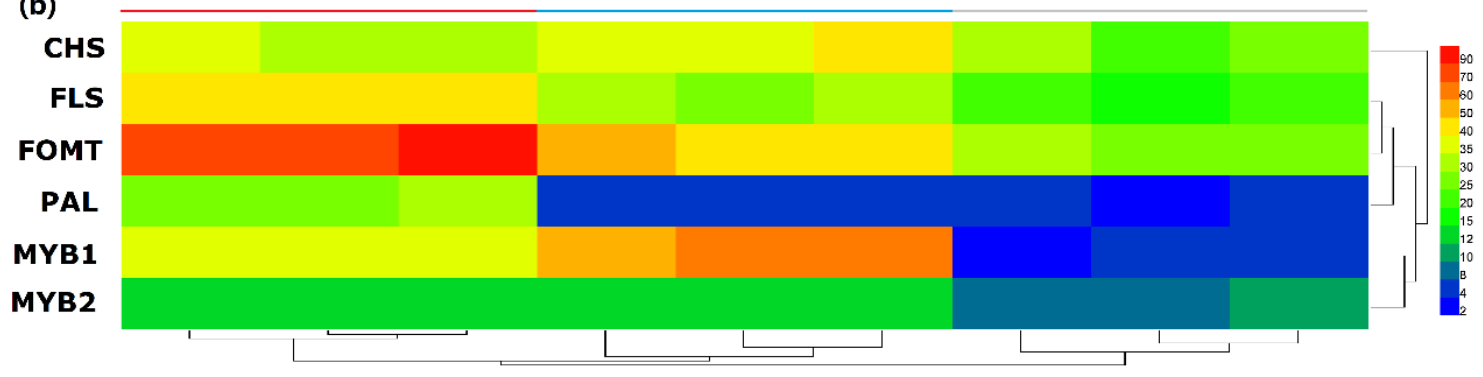

Fig. 9. The distribution of flavonoids and the transcription of related genes in Ginkgo leaves after treatmented with Se. (a) Heat map visualization of relative differences of flavonoids in leaves. Data of the content value of each flavonoid were normalized to complete linkage hierarchical clustering. A treatment with Se on leaves and B treatment with Se on root, which is visualized in a single column; each flavonoid is represented by a single row. Red indicates high abundance whereas low relative flavonoids are blue (color key scale above heat map). (b) Heat map of relative genes of flavonoids in leaves after treated with Se spraying on leaves and roots

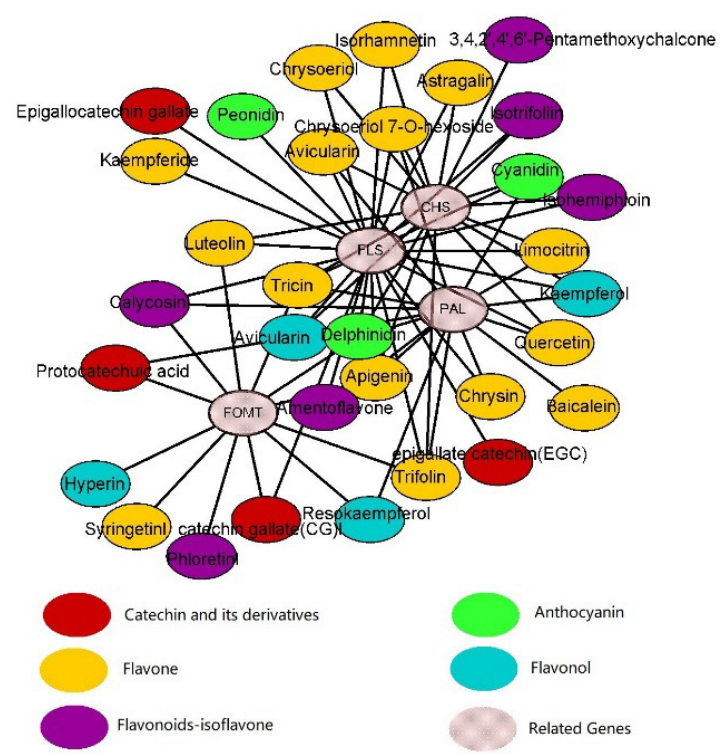

Fig. 10. Gene and related metabolites network diagram 

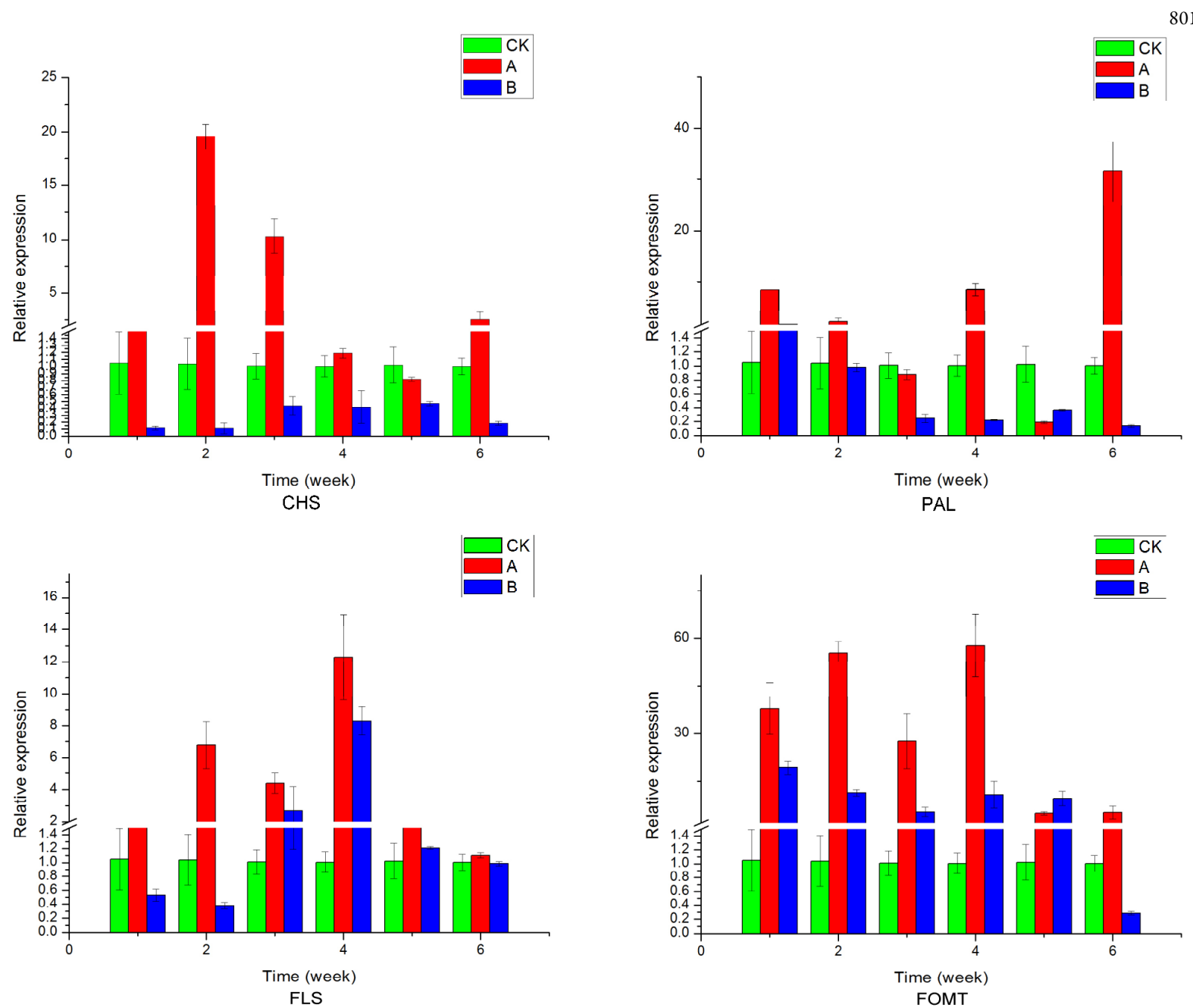

Fig. 10. Gene and related metabolites network diagram Fig. 11 qRT-PCR analysis of the transcript levels of four flavonoids genes after treatment with Se several weeks in Ginkgo leaves. Transcript levels are expressed relative to the expression of the GAPDH gene(Genbank accession no. L26924). Error bars represent the SD of three independent experiments. A. indicates leaves treatment spraying with Se; B. Root treatment with Se

kaempferol and a concomitant decrease of cinnamic acid derivatives. The tomato fruits can be safely enriched with $\mathrm{Se}$, and Se biofortification may enhance levels of other neutraceutical compounds (Schiavon et al., 2013). As for Gingko, Se with certain concentration can facilitate the chlorophyll synthesis, while excessive Se would hinder chlorophyll synthesis. Furthermore, Se process can effectively induce the accumulation of ginkgo leaves' quercetin, kaempferol and isorhamnetin (Chen, 2008).

In this research, we treatment the Ginkgo leaves and roots according to Chen' method (2008) describe, and then we classified the samples into three treatment groups (group A, group B, and the control group) to test their metabolome. According to the analytical results, flavanones of group A and B (e.g. vitexin, neohesperidin, apigenin, chrysin, catechinic, luteolin, homo-chrysoeriol) presented distinct increase than the control group. Besides, quercetin and kaempferol, as two metabolites of group B, also increased significantly. It can be concluded that $\mathrm{Se}$ in proper volume can facilitate the synthesis and accumulation of flavanones in Ginkgo leaves. The treatment of leaves presented obvious results, which is represented by the increase of various flavanones (e.g. proanthocyanidins, flavonoid-isoflavone, flavonoids, flavonoe-lignan, flavonol, flavonoid-C, flavone and anthocyanin).

\section{Influence of Se on ginkgo's genes relating to its secondary metabolism}

To investigate the influence of Se on ginkgo's specific genes relating to its synthesis of flavonoids, the four typical genes (CHS, FLS, FOMT, and PAL) and two MYB transcription factors concerning flavones metabolism were chosen from the transcriptome data for differential analysis of expression quantity.

Chalcone synthase (CHS) catalyses the first and key regulatory step of flavonoid biosynthetic pathway (Hahlbrock and Scheel, 1989). Correlation analysis between CHS activity and flavonoid accumulation during ginkgo leaf growth indicated that CHS might be the ratelimiting enzyme in the biosynthesis pathway of flavonoids in ginkgo leaves. Expression analysis showed that flavonoid accumulation paralleled the transcription level of change in 
802

chs gene, suggesting chs gene as the specific key gene regulating flavonoid accumulation in ginkgo leaves (Xu et al., 2007). Xu research showed that the transcription levels of GbPAL were significantly correlated with flavonoid accumulation, suggesting that GbPAL might play a regulatory role in flavonoid biosynthesis in leaves of $G$. biloba at the transcriptional level (Xu et al., 2008). Xu's research suggesting that GbFLS is a bifunctional enzyme within the flavonol biosynthetic pathway, GbFLS expression was induced by all of the six tested abiotic stresses, namely, UV-B, abscisic acid, cold, sucrose, salicylic acid, and ethephon (Xu et al., 2012). The GbFOMT gene expression level shows a correlation to the change of total flavonoid increase rate in the developmental stage of Ginkgo leaf. In this research, the transcription level of FLS and FOMT up regulated by treatment with Se in leaf and root, but the levels increased significantly in leaf, the expression results were more consistent with those of the metabolome data.

As shown by the results, applying Se fertilizer to ginkgo leaves can effectively improve the expression level of $\mathrm{CHS}$ and PAL, enhancing the content of flavonoids in ginkgo leaves. In contrast, Se treatment on roots didn't induce significant increase of CHS and PAL. Meanwhile, the contents of proanthocyanidins, and flavonoids-isoflavone in roots decreased. Se in certain concentration can improve the synthesis of chlorophyll and photosynthesis, thus effectively enhancing the contents of flavonoids in ginkgo (Chen, 2008; Feng et al., 2011). Therefore, Se treatment for leaves can stimulate photosynthesis and induce genetic expressions (CHS, FLS, FOMT, and PAL), which has a direct impact on flavonoids' accumulation in these tissues. However, high-concentration Se treatment may inhibit plants' growth and photosynthesis. In the case of Se treatment for roots, the root-top ratio would first increase and then decline, so did the biomass of leaves (Chen, 2008). Se treatment for roots in same concentration would lead to certain increase in FLS and FOMT genes in leaves but decrease in CHS and PAL genes, inhibiting the synthesis of flavonoids in leaves, which might be connected with the toxicity of Se to roots.

R2R3-MYB transcription factors (TFs) play key roles in the transcriptional regulation of the flavonoid biosynthesis in plants. Zhang's results suggested that GbMYBFL positively related to flavonoid biosynthesis, and the overexpression of GbMYBFL was sufficient to induce flavonoids and anthocyanin accumulation during $G$. biloba leaf growth, the transcription of GbMYBFL is positively correlated with the flavonoid content (Zhang et al., 2018). But the GbMYBF2 may have a key role in repressing transcription in regulating the biosynthesis of flavonoids in G. biloba (Xu et al., 2014a). In this research, The transcriptional levels of MYB1 in group A and group B are much higher than that of the control group, while MYB2 is a bit higher than the control group, indicating that transcription factor MYB1 and MYB2 are involved in positive regulation of $\mathrm{Se}$ treatment flavonoids accumulation. This is different from the negative regulation of R2R3MYB on ginkgo's flavonoids synthesis. It is obvious that $\mathrm{Se}$ treatment for leaves can regulate the accumulation of flavonoids by inducing flavonoids to synthesize related genetic expressions, while Se treatment for roots presents no significant difference.
The essentiality of Se for higher plants flavonoids metabolism has not been established to date, although there have been reports of beneficial effects of Se on plant growth of hyper-accumulators and, at low doses, of non-hyperaccumulator plants (Schiavon et al., 2013). Chen (2017) research results also suggesting that Se-containing polysaccharide induced apoptosis occurs through the mitochondria-dependent pathway in G. biloba. Se-GBLP therefore merits further investigation as a promising preventive and/or therapeutic agent against human bladder cancer. This study employ $G$. biloba leaves as material to explore the effect of $\mathrm{Se}$ on the synthesis of flavonoids compounds and key gene regulating mechanism with an attempt to provide theoretical and practical basis for increasing flavonoids content in $G$. biloba leaves.

\section{Acknowledgements}

This work was supported by the Natural Science Foundation of China (31400556), Economic Forest Germplasm Improvement and Comprehensive Utilization of Resources of Hubei Key Laboratories (2017AX02) and Scientific Research Key Project of Hubei Educational Office (D20162901).

\section{Conflicts of interest}

The authors declare that there are no conflicts of interest related to this article.

\section{References}

Beek TAV, Montoro P (2009). Chemical analysis and quality control of Ginkgo biloba leaves, extracts, and phytopharmaceuticals. Journal of Chromatography A 1216(11):2002-2032.

Beek TAV, Montoro P (2009). Chemical analysis and quality control of Ginkgo biloba leaves, extracts, and phytopharmaceuticals. Journal of Chromatography A 1216(11):2002-2032.

Bilia AR(2002). Ginkgo biloba L. Fitoterapia 73(3):276-279.

Chen W, Gong L, Guo Z, Wang W, Zhang H, Liu X, ... Luo J (2013). A novel integrated method tor large-scale detection, identification, and quantification of widely targeted metabolites: application in the study of rice metabolomics. Molecular Plant 6(6):1769-1780.

Chen D, Sun S, Cai D, Kong G (2017). Induction of mitochondrialdependent apoptosis in T24 cells by a selenium (Se)-containing polysaccharide from Ginkgo biloba L. leaves. International Journal of Biological Macromolecules 101:126-130.

Chen J (2008). Ginkgo physiological growth change response to selenium. Nanjing Forestry University, Place Nanjing Forestry University Published.

Cheng H, Li L, Cheng S, Cao F, Wang Y, Yuan H (2011). Molecular cloning and function assay of a chalcone isomerase gene $(\mathrm{GbCHI})$ from Ginkgo biloba. Plant Cell Reports 30(1):49-62.

Cheng H, Li L, Feng X, Yan W, Yuan H, Wu C, ... Wang Y (2013a). Expression patterns of an isoflavone reductase-like gene and its possible roles in secondary metabolism in Ginkgo biloba. Plant Cell Reports 32(5):637-650. 
Cheng H, LinlingL, Shuiyuan C, Fuliang C, Feng X, Honghui Y, Conghua W (2013b). Molecular cloning and characterization of three genes encoding dihydroflavonol-4-reductase from Ginkgo biloba in anthocyanin biosynthetic pathway. PloS One 8:e72017.

Feng X, Cheng SY, Zhu J, Zhang WW, Yan W (2011). Effects of 5aminolevulinic acid on chlorophyll, photosynthesis, soluble sugar and flavonoids of Ginkgo biloba. Notulae Botanicae Horti Agrobotanici Cluj-Napoca 39(1):41-47.

Fischer TC, Gosch C, Pfeiffer J, Halbwirth H, Halle C, Stich K, Forkmann $\mathrm{G}$ (2007). Flavonoid genes of pear (Pyrus communis). Trees 21(5):521529.

Grabherr MG, Haas BJ, Yassour M, Levin JZ, Thompson DA, Amit I, ... Zeng Q (2011). Full-length transcriptome assembly from RNA-Seq data without a reference genome. Nature Biotechnology 29(7):644.

Hahlbrock K, Scheel D (1989). Physiology and molecular biology of phenylpropanoid metabolism. Annual Review of Plant Biology 40(1):347-369.

Hua C, LinlingL, Feng X, Yan W, Honghui Y, Conghua W, ... Yuping W (2013). Expression patterns of an isoflavone reductase-like gene and its possible roles in secondary metabolism in Ginkgo biloba. Plant Cell Reports 32(5):637-650.

Jie C, Renke T, Xiaojuan G, Zhengii F, Zheng W, Zhiyan Z, Xiaoli T (2015). The functional annotation of unigenes in COG, GO, KEGG, Swissprot and Nrdatabase.

Kennedy DO, Wightman EL (2011). Herbal extracts and phytochemicals: plant secondary metabolites and the enhancement of human brain function. Advances in Nutrition 2(1):32-50.

Khan N, Adhami VM, Mukhtar H (2010). Apoptosis by dietary agents for prevention and treatment of prostate cancer. Endocrine Related Cancer 17(1):R39-R52.

Li LL, Cheng H, Yuan HH, Xu F, Cheng SY, Cao FL (2014). Functional characterization of the Ginkgo biloba chalcone synthase gene promoter in transgenic tobacco. Genetics \& Molecular Research 13(2):34463460.

Pang Y, Shen G, Wu W, Liu X, Lin J, Tan F, ... Tang K (2005). Characterization and expression of chalcone synthase gene from Ginkgo biloba.Plant Science 168(6):1525-1531.

Qian L, Liu Y, Qi Y, Jiao S, Tian F, JiangL, Wang Y (2014). Transcriptome sequencing and metabolite analysis reveals the role of delphinidin metabolism in flower colour in grape hyacinth. Journal of Experimental Botany 65(12):3157-3164.

Schiavon M, Dall'Acqua S, Mietto A, Pilonsmits EA, Sambo P, Masi A, Malagoli M (2013). Selenium fertilization alters the chemical composition and antioxidant constituents of tomato (Solanum lycopersicon L.). Journal of Agricultural \& Food Chemistry 61:1054210554 .
Schmittgen TD, Livak KJ (2008). Analyzing real-time PCR data by the comparative C(T) method. Nature Protocols 3(6):1101.

Thavarajah D, Ruszkowski J, Vandenberg A (2008). High potential for selenium biofortification of lentils (Lens culinaris L.). Journal of Agricultural and Food Chemistry 56(22):10747-10753.

Tian XY, Wang ZY (2008). Effects of selenium application on content, distribution and accumulation of selenium, flavonoids and rutin in tartary buckwheat. Plant Nutrition \& Fertilizer Science 14(4):721-727.

Winkel-Shirley B (2002). Biosynthesis of flavonoids and effects of stress. Current Opinion in Plant Biology 5(3):218-223.

Xu F, Cai R, Cheng S, Du H, Wang Y (2008). Molecular cloning, characterization and expression of phenylalanine ammonia-lyase gene from Ginkgo biloba. African Journal of Biotechnology 7(6):721-729.

XuF, ChengS, Cheng S, Wang Y, Du H (2007). Time course of expression of chalcone synthase gene in Ginkgo biloba. Journal of Plant Physiology and Molecular Biology 33(4):309.

Xu F, Li L, Zhang W, Cheng H, Sun N, Cheng S, Wang Y (2012). Isolation, characterization, and function analysis of a flavonol synthase gene from Ginkgo biloba. Molecular Biology Reports 39(3):2285-2296.

Xu F, Ning Y, Zhang W, Liao Y, Li L, Cheng H, Cheng S (2014a). An R2R3-MYB transcription factor as a negative regulator of the flavonoid biosynthesis pathway in Ginkgo biloba. Functional \& Integrative Genomics 14(1):177-189.

Xu Q, Yin XR, Zeng JK, Ge H, Song M, Xu CJ, ... Chen KS (2014b). Activator- and repressor-type MYB transcription factors are involved in chilling injury induced flesh lignification in loquat via their interactions with the phenylpropanoid pathway. Journal of Experimental Botany 65(15):4349-4359.

Ye J, Chen Q, Tao T, Xu F, Cheng S (2016). Promotive effects of genistein on photosynthesis, flavonoid biosynthesis and antioxidant enzyme activities in Ginkgo biloba. International Journal of Agriculture and Biology 18(5):917-922.

Zhai R, WangZ,ZhangS, Meng G, SongL, WangZ,...XuL (2016). Two MYB transcription factors regulate flavonoid biosynthesis in pear fruit (Pyrus bretschneideri Rehd.). Journal of Experimental Botany 67(5):1275-1284.

Zhang W, Xu F, Cheng S, Liao Y (2018). Characterization and functional analysis of a MYB gene (GbMYBFL) related to flavonoid accumulation in Ginkgo biloba. Genes \& Genomics 40(1):49-61. 